\title{
Contrast Enhancement Using Brightness Preserving Histogram Equalization Technique for Classification of Date Varieties
}

\author{
G Thomasa, A Manickavasagan*b, L Khrijic and R Al-Yahyaid \\ a Department of Electrical and Computer Engineering, Faculty of Engineering, University of Manitoba, Canada \\ ${ }^{* b}$ Department of Soils, Water and Agricultural Engineering, College of Agricultural and Marine Sciences, Sultan Qaboos \\ University, Al-Khoud, Muscat, Sultanate of Oman \\ ${ }^{c}$ Department of Electrical and Computer Engineering, College of Engineering, Sultan Qaboos University, Al-Khoud, \\ Muscat, Sultanate of Oman \\ d Deepartment of Crop Science, College of Agricultural and Marine Sciences, Sultan Qaboos University, Al-Khoud, Muscat, \\ Sultanate of Oman
}

Received 5 January 2013; accepted 11 December 2013

\begin{abstract}
Computer vision technique is becoming popular for quality assessment of many products in food industries. Image enhancement is the first step in analyzing the images in order to obtain detailed information for the determination of quality. In this study, Brightness preserving histogram equalization technique was used to enhance the features of gray scale images to classify three date varieties (Khalas, Fard and Madina). Mean, entropy, kurtosis and skewness features were extracted from the original and enhanced images. Mean and entropy from original images and kurtosis from the enhanced images were selected based on Lukka's feature selection approach. An overall classification efficiency of $93.72 \%$ was achieved with just three features. Brightness preserving histogram equalization technique has great potential to improve the classification in various quality attributes of food and agricultural products with minimum features.
\end{abstract}

Keywords: Computer vision, Brightness preserving histogram equalization, Dates variety.

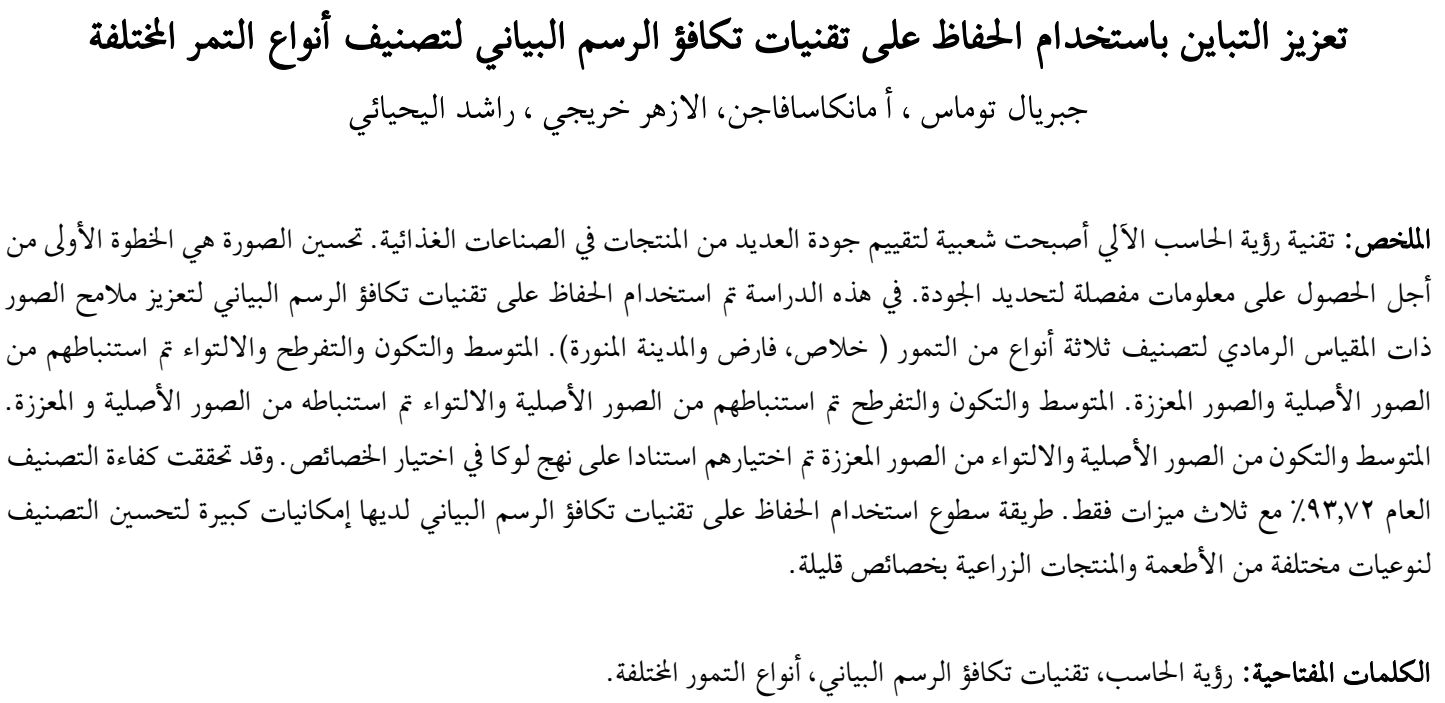

$\overline{\text { *Corresponding author's e-mail: manick@squ.edu.om }}$ 


\section{Introduction}

Date is an important agricultural commodity in Oman. Around $50 \%$ of the total cultivated land in Oman is under date palm vegetation. Although production of dates has been improved from 45,000 Mt in 1970 to 255,891 Mt in 2007 (FAO 2007), quality has been a major concern in Oman. Quality, composition and packaging have always been a problem for Omani dates to compete in the international market (AlMarshudi 2002). The average annual export is only 9000 metric tons which is 2.5 to $3.5 \%$ of production (FAO 2007). Whereas in some other date producing countries, a major portion of the production is being exported. For example, 55.5\% in Tunisia, 54.7\% in Israel, $24.2 \%$ in Iran and $18.7 \%$ in Pakistan are exported from their production (FAO 2007). Varietal purity, color, uniformity of size and absence of defects are some of the important quality parameters for dates in domestic and international markets. In general, manual grading of dates is followed in handling and processing facilities. This method has many constraints such as subjectivity (personal perception), influence of mental stress, influence of environment (lightings), efficiency of individuals at various times of the shift and so on (Brosnan and Sun 2002).

Computer vision technology is becoming popular in the evaluation of various qualities of agricultural and food products. In external quality evaluation, visual attributes such as the shape, size, color, and surface texture are analyzed from the captured images. Chemical components such as starch, protein, oil, sugar, and moisture are analyzed with the help of unique imaging systems (Manickavasagan and AlYahyai, 2012). In North America, the food sector is one of the top 10 industries using computer vision technology (Gunasekaran 1996). Computer vision systems using color cameras have wide application in grading and quality evaluation of food materials based on visual color characteristics. In the handling and processing of fruits, evaluation of bicolor fruit in mango (Kang et al. 2008), grading of strawberry (Liming and Yanchao 2010), grading of apple (Leemans et al. 2002), sorting of Satsuma mandarin (Blasco et al. 2009), quality evaluation of orange (Kondo et al. 2000) and peel defects in citrus (Blasco et al. 2007) are some applications that have used color cameras. Manickavasagan et al. (2008) developed a monochrome imaging system to identify eight wheat classes.

Fadel et al. (2006) measured the color properties of five date varieties (Lolo, Khalas, Berhi, Fard, and Bomaan) from UAE to develop classification protocols. The RGB color values were analyzed for the test ed varieties. It was determined that the $\mathrm{R}$ component was suitable for discrimination of Berhi from Bomann and Berhi from Fard, the $\mathrm{G}$ component was good for the classification of Fard and Berhi, and Fard and Bomann. Similarly, the B component was suitable for differentiating Fard and Berhi; Fard and Bomaan; Lolo and Berhi; and Khalas and Berhi. Lee et al. (2008a) developed a color space conversion method and color index distribution analysis technique for the automated evaluation of date maturity. An overall accuracy of about $90 \%$ was achieved while identifying seven grades based on the maturity level. Fadel (2008) developed algorithms to determine sugar content of dates (Lolo and Bomoaan varieties) based on color analysis. RGB components of the images of the date samples acquired with a CCD color camera were correlated with actual values of fructose and glucose measured by HPLC. It was determined that a minimum accuracy of $86 \%$ was achieved while determining the sugar content of dates using RGB components. Lee et al. (2008b) developed a machine vision system for automatic date grading using reflective NIR imaging. The surface reflectance of the date fruit was depending on the skin delamination level. The percentage of delamination was calculated and used in grade determination. The overall accuracy of the system was in the range of $79-95 \%$ for various grades.

The efficiency of edge detection features in classifying dates based on hardness was determined by Manickavasagan et al. (2013). The overall classification accuracies were $75 \%$ and $87 \%$ while using ANN for three-class (soft, semihard and hard) and two-class (soft and hard (semi-hard and hard together)) models, respectively. Al-Rahbi et al. (2013) used RGB color imaging technique to classify three grades of dates based on surface crack (no-crack dates, low-crack dates and high-crack dates). In three classes model, the classification accuracy was $62 \%, 58 \%$ and $78 \%$ for high-crack, low-crack and no-crack dates, respectively using linear discriminant analysis (LDA).

Although computer vision based inspection has been used for various quality measurements with acceptable accuracy in many food industries, algorithm development and training of calibration models are the important components in determining their accuracy. Automated inspection procedures for the quality assurance will provide great opportunity for Omani dates to compete in the international market. Therefore the objective of this study was to determine the classification of three date varieties using gray scale images and the brightness preserving histogram equalization enhancement technique. 


\section{Materials and Methods}

\subsection{Image Acquisition}

Three date varieties namely Khalas, Fard and Madina were used in this study. Samples for each variety were obtained from at least three shops in Oman, and the varietal purity was confirmed by a "date variety expert" at Sultan Qaboos University. A conglomerate sample of 108 dates was taken for each variety $(n=108$ for each variety) and the sample was imaged (single date images) with a color camera (model: D3, Nikon, Japan, Resolution - $4256 \times 2832$ pixels). The date samples were illuminated with halogen lamp (Visa tech, model SOLO 1600B) during imaging. Then all images were converted into gray scale images using Matlab software, and analyzed. A threshold operation was done to eliminate the background based on the maximization of the between-class variance using Otsu's method (Otsu 1979).

\subsection{Brightness Preserving Histogram Equiliza- tion}

In this section the image enhancement technique which preserves the mean of the original image is presented. Even though, it was originally introduced as an image enhancement technique (Wang and Ye, $2005)$, it is used here as a way to enhance the classification features. Because the mean value of an image is associated with its brightness, this approach was named Brightness Preserving Histogram Equalization (BPHE). As the name indicates, the method relies on histogram modification and this section starts with a brief discussion on Histogram Equalization (HE), a common and popular contrast enhancement technique. We included a brief discussion here but more detail can be found in Wang and Ye (2005).

It is well known that certain images can cause problems when using HE (Gonzalez and Woods 2001). In some cases, the histogram of an image shows a strong peak because the image is dominated by a large area of a single gray level value. Regardless of the shape of the histogram in the original image, HE will yield an image with a final level of brightness that is close to 0.5 .

Wang and Ye (2005) proposed a technique that yields an image with a similar brightness level as the original one. The idea is to find a specified histogram $f_{Z}(z)$ which mean or average level of brightness is equal to the original one subject to the constraint that the entropy is maximum. Because the uniform distribution has maximum entropy, this condition offers excellent contrast.

Mathematically the method is expressed as:

$$
\max _{\mathrm{f}}\left\{-\mathrm{f}_{Z}(\mathrm{z}) \ln \mathrm{f}_{Z}(\mathrm{z}) \mathrm{dz}\right\} \text {, s.t. }\left\{\begin{array}{c}
\mathrm{f}_{Z}(\mathrm{z}) \geq 0 \\
\int_{0}^{1} \mathrm{f}_{Z}(\mathrm{z}) \mathrm{dz}=1 \\
\int_{0}^{1} \mathrm{zf} \mathrm{f}_{Z}(\mathrm{z}) \mathrm{dz}=\mu_{\mathrm{o}}
\end{array}\right.
$$

$$
\text { for } z \in[0,1] \text { and } \mu_{o}=\int_{0}^{1} r f_{R}(r) d r \text {. }
$$

A functional can be formed as:

$$
\begin{aligned}
& J\left(f_{Z}(z)\right)=-\int_{0}^{1} f_{Z}(z) \ln f_{Z}(z) d z \\
& +\lambda_{1}\left[\int_{0}^{1} f_{Z}(z) d z-1\right]+\lambda_{2}\left[\int_{0}^{1} z f_{Z}(z) d z-\mu_{o}\right]
\end{aligned}
$$

Where $\lambda_{1}$ and $\lambda_{2}$ are Lagrange multipliers associated with the constraints in (1). This is solved using calculus of variations:

$$
\frac{\partial \mathrm{J}}{\partial \mathrm{f}_{\mathrm{Z}}(\mathrm{z})}=-\ln \mathrm{f}_{\mathrm{Z}}(\mathrm{z})-1+\lambda_{1}+\lambda_{2} \mathrm{z}
$$

yielding:

$$
f_{Z}(z)=e^{\mu_{1}-1} e^{\lambda_{2} z} \text { for } z \in[0,1]
$$

Using the constraints in (1):

$$
f_{Z}(z)=\left\{\begin{array}{cc}
1 & \text { if } \mu_{0}=0.5 \\
\frac{\lambda_{2} e^{\lambda_{2}}}{e^{\lambda_{2}}-1} & \text { if } \mu_{0} \in(0,0.5) \cup(0.5,1)
\end{array}\right.
$$

once again for $z \in[0,1]$.

The second Lagrange multiplier can be found as $\mu_{\mathrm{o}}=\int_{0}^{1} \mathrm{zf}_{\mathrm{Z}}(\mathrm{z}) \mathrm{dz}$ yielding: $\mu_{\mathrm{o}}=\frac{\lambda_{2} \mathrm{e}^{\lambda_{2}}-\mathrm{e}^{\lambda_{2}}+1}{\lambda_{2}\left(\mathrm{e}^{\lambda_{2}}-1\right)}$

which can be found using a lookup table as suggested in wang and Ye (2005). When BPHE is applied to the original images, rather than expecting nice images we are looking for more contrast but preserving the level of darkness of the original ones.

\section{Results and Discussion}

Figure 1 shows the original gray scale images of the date varieties. Starting by considering an image with background class defined as $w_{b}$, and object in class $w_{o}$, with probabilities $p\left(w_{b}\right)=\sum_{i=1}^{T-1} f\left(z_{i}\right)$ and $p\left(w_{o}\right)=\sum_{i=T}^{L} f\left(z_{i}\right)$ where $z$ denotes discrete image intensity, $f\left(z_{i}\right), i=1,2, \ldots, L$ is the corresponding histogram normalized to have area equal to one, and $L$ is the number of distinct intensity levels, the threshold $T$ is found by maximizing: 

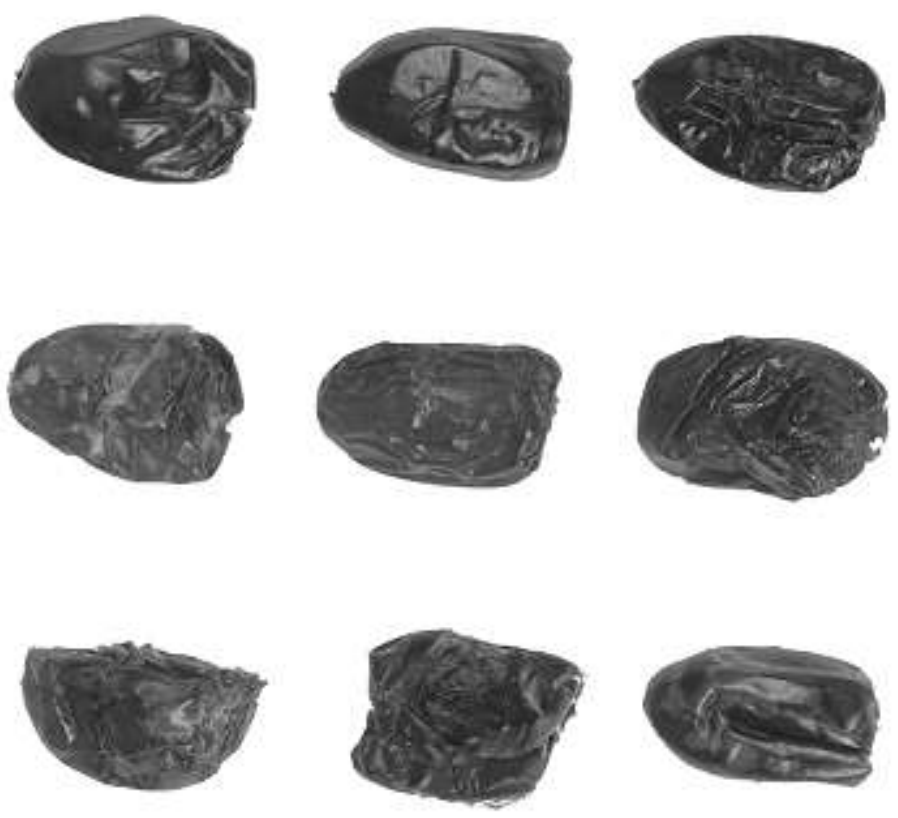

Figure 1. Original images of date varieties (first row Fard, second row Madina, third row Khalas).

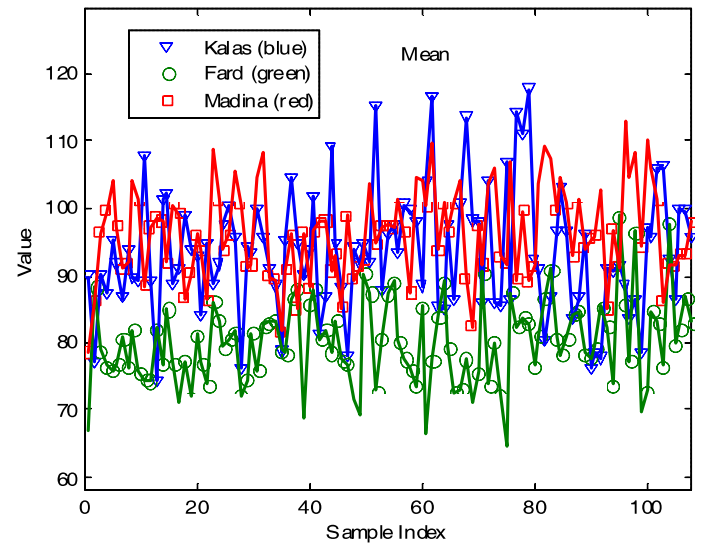

(a) Mean gray level values

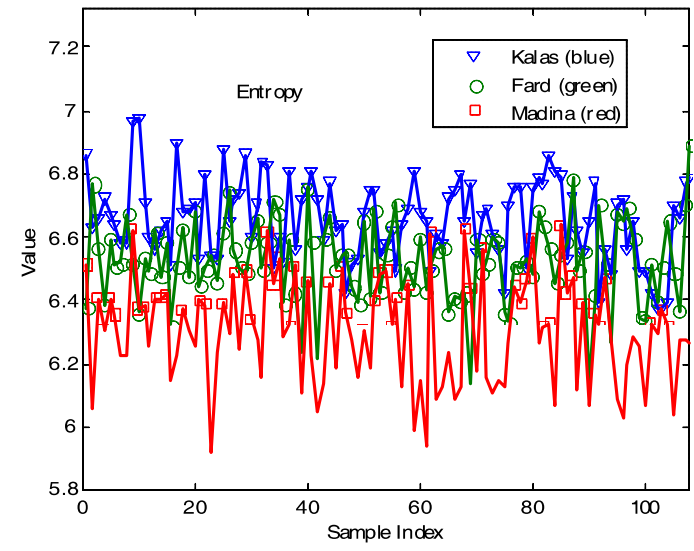

(b) Entropy values

Figure 2. Mean gray values and entropy values of dates.

$$
\sigma_{B}^{2}(T)=p\left(w_{b}\right)\left(\mu_{b}-\mu_{t o t}\right)^{2}+p\left(w_{o}\right)\left(\mu_{o}-\mu_{t o t}\right)^{2}
$$

Where $\mu_{b}=\sum_{i=1}^{T-1} Z_{i} f\left(Z_{i}\right), \mu_{o}=\sum_{T}^{L} Z_{i} f\left(Z_{i}\right)$ and

$$
\mu_{\text {tot }}=\sum_{i=1}^{L} z_{i} f\left(Z_{i}\right)
$$

From the small image sample shown in Fig. 1, it can be inferred that features such as the mean value would work for the class Fard, since they look darker than the other two classes. Figure $2 \mathrm{a}$ shows the mean values $m$ for 108 images of each class calculated with the same expression above so that $m=\mu_{t o t}$. Note how in Fig. 2a the values for class Fard do appear darker and dissimilar than the other two.
Another feature that can be calculated to help the classification is entropy defined by Shannon (1948) as:

$$
H=\sum_{i=1}^{L} f\left(Z_{i}\right) \log \left(f\left(Z_{i}\right)\right)
$$

Entropy captures texture information and measures dispersion of these histograms. Entropy values can be seen in Fig. 2b. Figure 2a suggests that the mean values can help on classifying the images corresponding to the Fard class, and Fig. $2 \mathrm{~b}$ indicates the possible usefulness of including the entropy values in a classifier for discriminating the Madina class. The scatter plot in Fig. 3 shows how these two features may not be enough since the clusters for each class overlap considerably.

In order to also consider the shape of the his- 


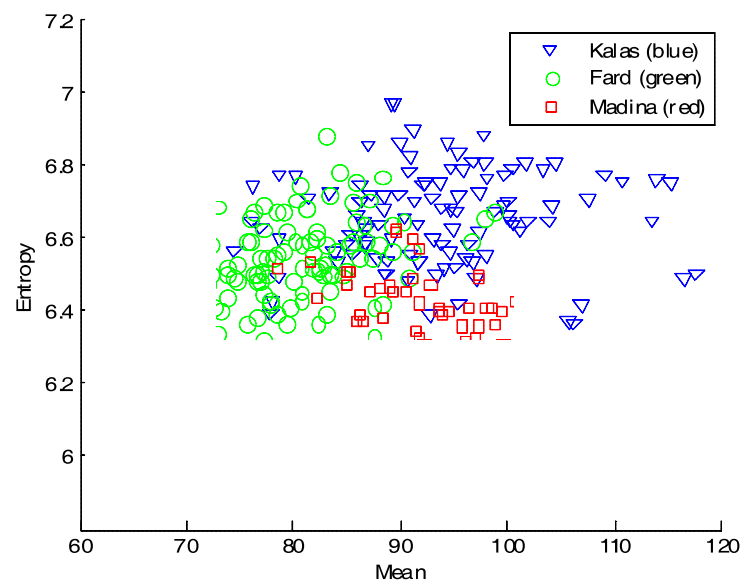

Figure 3. Scatter plot of mean and entropy value of dates.

tograms, other statistical features can be introduced in the form of standardized moments defined by Kenney and Keeping (1962):

$$
\gamma=\frac{\mu_{n}}{\sigma^{n}}=\frac{E[z-m]^{n}}{\sigma^{n}}
$$

Where $E$ is the expected value, $\sigma$ the standard deviation and $\mu_{n}$ is the nth moment about the mean. For $n=3$ one can find what is known as skewness that measures the asymmetry of the probability density function of the random variable associated to the image values. These skewness values were calculated for the entire image set and are shown in Fig. 4. For $n=4$ the value is known as kurtosis which also evaluates the shape of a distribution by quantifying its peakness/flatness.

From the values calculated, this last feature can help to distinguish the last class, Khalas, and a simple classifier may do the job when using only these three features. For this reason, a Bayes classifier for Gaussian pattern classes under the condition of a 0-1 loss function was implemented forming three decision functions $d_{j}(\mathbf{x})$ for $j=1,2,3$ in the form:

$d_{j}(\mathbf{x})=\ln P\left(w_{j}\right)-(1 / 2) \ln \left|\mathbf{C}_{j}\right|-(1 / 2)\left[\left(\mathbf{x}-\mathbf{m}_{j}\right)^{T} \mathbf{C}_{j}^{-1}(\mathbf{x}-\right.$ $\left.\mathbf{m}_{j}\right)$ ]

where $\mathbf{m}_{j}=E_{j}\{\mathbf{x}\}$ are the means of the feature grouped in vector $\mathbf{x}$, and $\mathbf{C}_{j}=E_{j}\left\{\left(\mathbf{x}-\mathbf{m}_{j}\right)\left(\mathbf{x}-\mathbf{m}_{j}\right)^{T}\right\}$ (Gonzalez and Woods, 2001).

This classifier was tested using 80 samples for training and 28 samples for testing. A $72.62 \%$ of correct classifications was obtained.

To improve on these preliminary results, without going for an extensive search of different features, we decided for enhancing the images not for visual purposes, but rather to enhance the features already

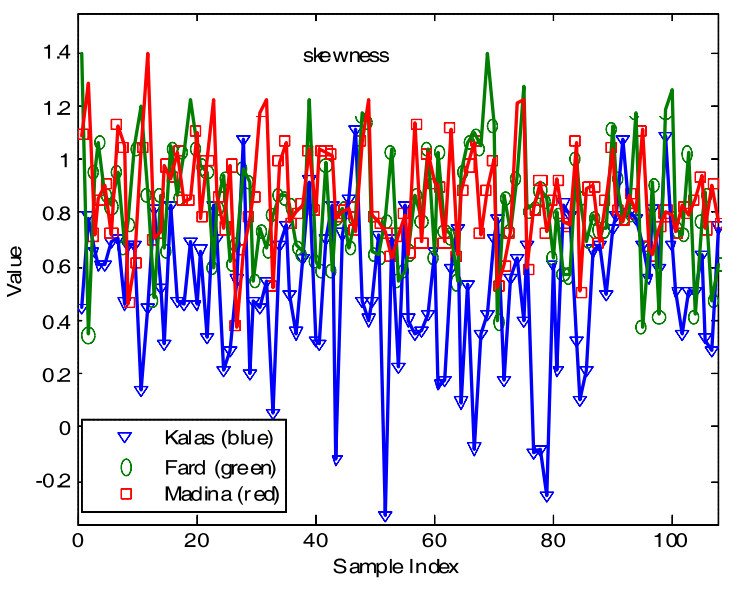

Figure 4. Skewness values of dates.

obtained. Thus, the following section will describe an innovative way to alter the features in such a way to improve the classification. The mean value was a feature to keep because a quick visual inspection of all the images provided a good clue on their importance that was further corroborated by the plots shown in Fig. 2a. The images were enhanced using the BPHE technique that improves contrast without modifying the mean values. The classifier was to be changed to a neural network to improve on the results obtained by the Bayes classifier. The image enhancement technique by BPHE is parameter free and quite fast, both desirable characteristics to have in order for an operator to avoid any necessary additional training. Thus, only the number of neurons in the neural network is to be used as a parameter for any possible future adjustments to the proposed system.

\subsection{Evaluation of Features Obatined from Ima- ges Enhaced by BPHE}

Figure 5 shows the enhanced image of the date samples in three varieties. Figure 6 shows the kurtosis and entropy values for the original set of images and the contrast enhanced ones corroborating these changes. As indicated before, these changes happen without modifying the original values for the mean. With these results in mind, the following section starts by justifying the use from such modified images to be used as inputs to a neural network based classifier. To validate the use of the image enhancement technique, a feature selection approach was used to compare the features extracted with and without the contrast enhancement. Because of the results already obtained using the Bayes classifier with just three features, it was contemplated to use only a much reduced number of features. This will in turn make the training of the neural network classifier fast given the enhanced quality of the features. Then, for the feature evaluation, a selection scheme with a backward sequential search starting 

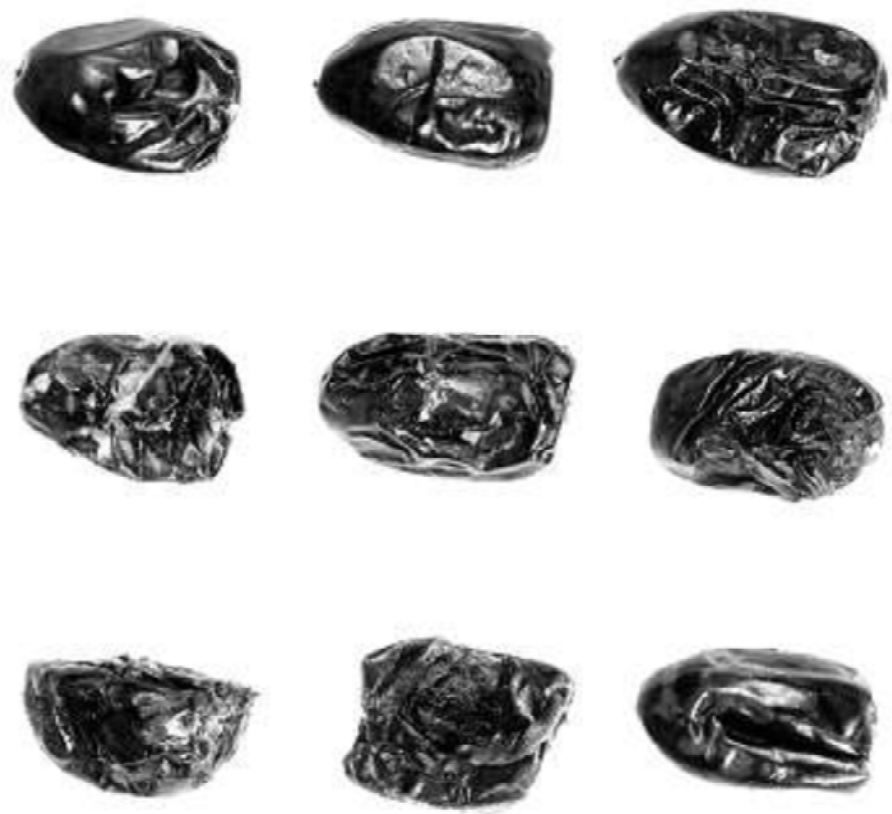

Figure 5. Enhanced images of date samples with BPHE technique (first row Fard, second row Madina, third row Khalas).

with a full feature set and sequentially removing features was considered to be a practical solution. We decided to use a new feature selection scheme that is based on fuzzy entropy measures with a similarity classifier as presented in Luukka (2001) which is briefly described next.

The method starts by forming an ideal vector $\mathbf{v}_{i}=$ $\left(v_{i}\left(f_{1}\right), v_{i}\left(f_{2}\right), \ldots, v_{i}\left(f_{\mathrm{t}}\right)\right)$ that represents the class $i$ as good as possible by calculating the mean values of available vectors in each class $v_{i}\left(f_{i}\right)$ for $i=1,2, \ldots \mathrm{t}$ features. Afterwards, the similarities $\mathbf{S}(\mathbf{x}, \mathbf{v})$ between the samplex ' $\mathrm{x}$ ' to be classified and the ideal vectors $\mathbf{v}$ need to be calculated as:

$$
S(x, v)=\frac{1}{t} \sum_{r=1}^{t}\left(1-\left|x\left(f_{r}\right)-v\left(f_{r}\right)\right|\right)
$$

Where $\mathrm{t}=$ total number of features;

In order to calculate the relevance of the features, fuzzy entropy values are calculated with similarity values $\mu_{A}\left(x_{j}\right)$ as suggested by Luca and Termini (1971):

$$
H_{1}(A)=-\sum_{j=1}^{n}\left(\begin{array}{c}
\mu_{A}\left(x_{j}\right) \log \mu_{A}\left(x_{j}\right)+\left(1-\mu_{A}\left(x_{j}\right)\right) \\
\log \left(1-\mu_{A}\left(x_{j}\right)\right)
\end{array}\right)
$$

Where $\mathrm{n}=$ total number of classes;

The low entropy indicates high similarity values and high entropy values are obtained otherwise. Similarly, fuzzy entropy as suggested by Parkash et al. (2008) was used in this work which follows the expression:

$$
H_{2}(A)=-\sum_{j=1}^{n}\left(\sin \frac{\pi \mu_{A}\left(x_{j}\right)}{2}+\sin \frac{\pi\left(1-\mu_{A}\left(x_{j}\right)\right)}{2}-1\right)
$$

Mean, Shanon's entropy, kurtosis and skewness were evaluated together with the values of kurtosis and skewness computed from the contrast enhanced images. Feature selection by the method proposed by Luukka (2011) using both fuzzy entropy metrics resulted on favoring the use of kurtosis over the skewness values regardless of the type of image used, either original ones or the ones enhanced by BPHE. The kurtosis values selected were the ones using BPHE. This gives us justification for the use of these features in the neural network implemented and described in the following section.

\subsection{Neural Network and Classification Results}

A two layer feed-forward back propagation neural network was used updating the weight and bias values according to the Levenberg-Marquardt optimization training algorithm with hyperbolic tangent sigmoid transfer functions using Matlab as the software platform for development. The training algorithm used was the scaled conjugate gradient. The features to be used were the ones suggested by Luukka's feature selection approach: mean and Shannon's entropy values using the original images, and the Kurtosis values from the BPHE enhanced images. After a few trials changing the number of hidden neurons using 10, 20, 30 and 40, we found that the best network was the one with 10 neurons that yielded a correct classification of 


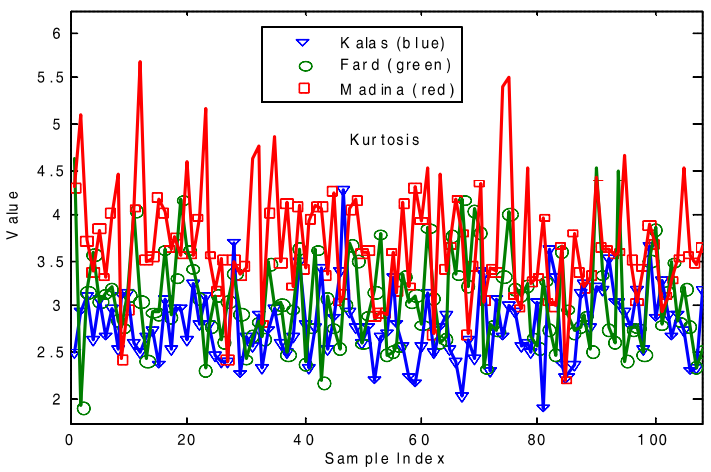

(a) Kurtosis from original images.

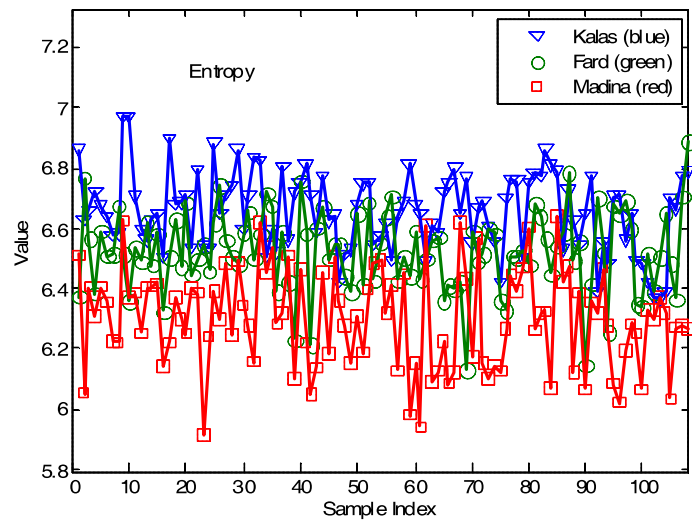

(c) Entropy from original images.

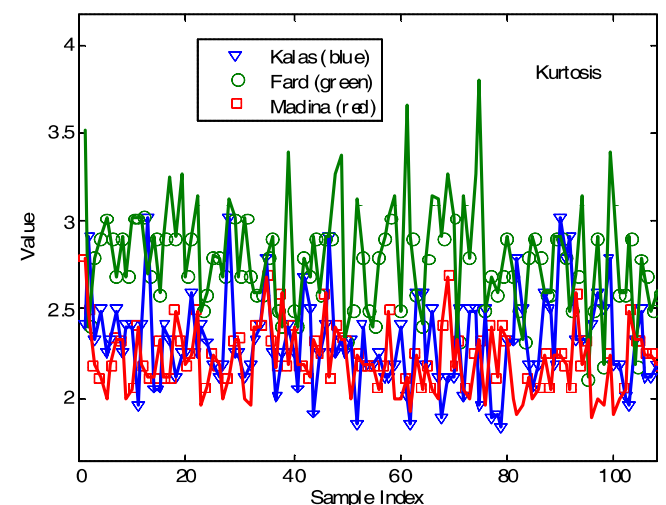

(b) Kurtosis from BPHE enhanced images.

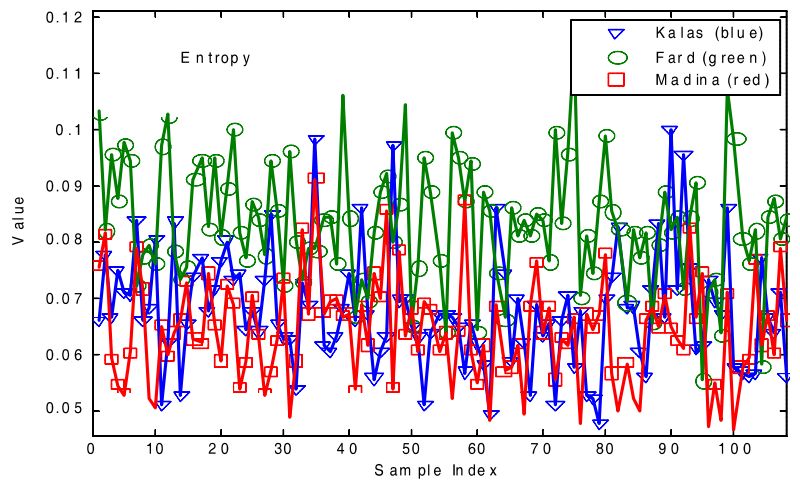

(d) Entropy from BPHE enhanced images.

Figure 6. Kurtosis and entropy of date varieties before and after BPHE enhancement.

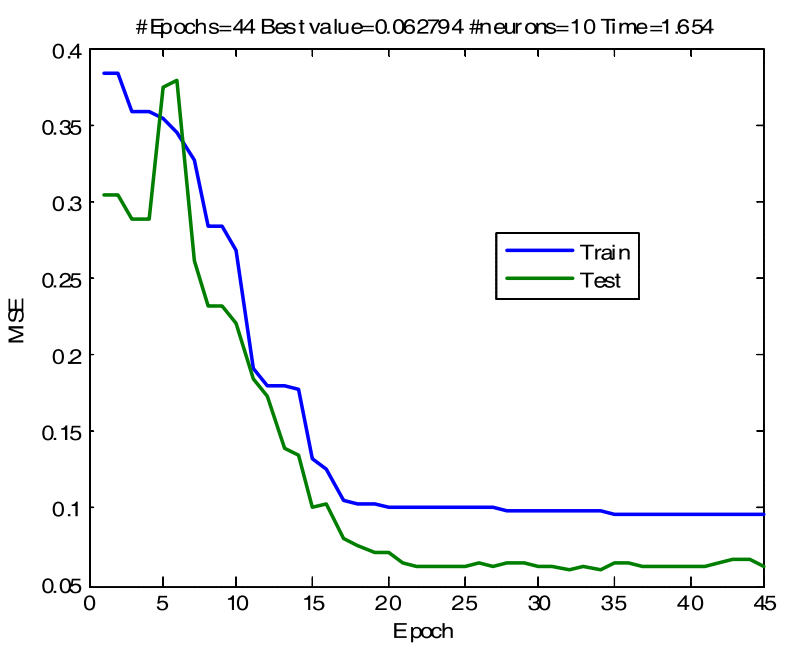

Figure 7. Mean squared error during neural network classification with features.

$93.72 \%$. This happened at epoch 38 and the training took 45 epochs in total in a total training time of only 1.654 seconds using an Asus Ee Slate tablet computer with an Intel i5 $1.33 \mathrm{GHz}$ processor and $4 \mathrm{~GB}$ of RAM memory. Figure 7 shows the plot of the mean squared errors calculated for the training and testing sets. A total of 48 images were used for testing and 60 images were used for training from each type of date $(55 \%$ Training: 45\% Testing).

To confirm the use of the BPHE method, neural networks were trained 30 times with different numbers of hidden neurons. The initial weights are calculated randomly by Matlab and these averages would give an idea of the better performance of the enhanced kurtosis feature. Because only three features are used, the expectation was that the network would not need many neurons. Table I shows the mean squared error results obtained while using the features extracted from original and BPHE enhancement separately.

\section{Conclusions}

The mean squared error (MSE) was reduced while using the features extracted from BPHE enhanced images in the ANN than from the original images. BPHE has great potential to enhance the features and improve the classification in food and agricultural products. In this study the gray scale images were enhanced and features were extracted. The enhancement of features in RGB color images and their effect in classification of dates in handling facilities must be studied. 
Table 1. Mean squared error during neural network classification with features from original and BPHE enhanced images separately while using different number of hidden neurons.

\begin{tabular}{lllllllll}
\hline & \multicolumn{2}{c}{$\mathbf{N n}^{*}=\mathbf{5}$} & \multicolumn{2}{c}{$\mathbf{N n}=\mathbf{1 0}$} & \multicolumn{2}{c}{$\mathbf{N n}=\mathbf{2 0}$} & \multicolumn{2}{c}{$\mathbf{N n}=\mathbf{3 0}$} \\
& BPHE & Original & BPHE & Original & BPHE & Original & BPHE & Original \\
\hline MSE \% & 11.52 & 13.00 & 11.78 & 13.48 & 12.17 & 13.17 & 13.81 & 14.46 \\
Time seconds & 0.8698 & 0.9344 & 1.0417 & 0.9792 & 0.9365 & 1.055 & 0.9557 & 0.9849 \\
\hline
\end{tabular}

\section{Acknowledgments}

We thank The Research Council (TRC) of Sultanate of Oman for funding this study (Project No. RC/AGR/SWAE/11/01-Development of Computer Vision Technology for Quality Assessment of Dates in Oman).

\section{References}

Al-Marshudi AS (2002), Oman traditional date palms: Production and improvement of date palms in Oman. Tropicultura 20:203-209.

Al-Rahbi S, Manickavasagan A, Al-Yahyai R, Khriji L, Alahakoon P (2013), Detecting surface cracks on dates using color imaging technique. Food Science and Technology Research 19:1-10.

Blasco J, Aleixos N, Molto E (2007), Computer vision detection of peel defects in citrus by means of a region oriented segmentation algorithm. Journal of Food Engineering 81:535-543.

Blasco J, Aleixos N, Cuero S, Comez-Sanchis J, Molto E (2009), Automatic sorting of (Citrus unshiu) segments using computer vision and morphological features. Computers and Electronics in Agriculture 66:1-8.

Brosnan T, Sun D (2002), Inspection and grading of agricultural and food products by computer vision systems - a review. Computers and Electronics in Agriculture 36(2):193-213.

Fadel M, Kurmestegy L, Rashed M, Rashed Z (2006), Fruit color properties of different cultivars of dates (Phoenix dactylifera, L.). Agricultural Engineering International-CIGR Journal VIII:1-9.

Fadel MA (2008), Sugar content estimation of date (Phoenix dactylifera, L.) fruits in tamr stage. Agricultural Engineering International-CIGR Journal X:1-9.

FAO (2007), FAO Statistics. Available at: http://faostat.fao.org/site/342/default.aspx. Accessed on February 24, 2013.

Gonzalez RC, Woods RE (2001), Digital Image Processing, Prentice Hall, second edition, NJ, USA.

Gunasekaran S (1996), Computer vision technology for food quality assurance. Trends in Food Science and Technology 7:245-256.
Kang SP, East AR, Trujillo FJ (2008), Colour vision system evaluation of bicolour fruit: A case study with 'B74' mango. Postharvest Biology and Technology 49:77-85.

Kenney JF, Keeping ES (1962), Moments in standard units. Mathematics of Statistics, Pt. 1, 3rd ed. Van Nostrand, Princeton, NJ, USA.

Kondo N, Ahmad U, Monta M, Murase H (2000), Machine vision based quality evaluation of Iyokan orange fruit using neural networks. Computers and Electronics in Agriculture 29:135-147.

Liming X, Yanchao Z (2010). Automated strawberry grading system based on image processing. Computer and Electronics in Agriculture 71:3239.

Lee D, Archibald JK, Chang Y, Greco R (2008a), Robust color space conversion and color distribution analysis techniques for date maturity evaluation. Journal of Food Engineering 88:364-372.

Lee D, Schoenberger R, Archibald J, McCollum S (2008b), Development of machine vision system for automatic date grading using digital reflective near-infrared imaging. Journal of Food Engineering 88:388-398.

Leemans V, Magein H, Destain MF (2002), On-line fruit grading according to their external quality using machine vision. Biosystems Engineering 84:397-404.

Luca D, Termini S (1971), A definition of non-probabilistic entropy in setting of fuzzy set theory. Information Control 20:301-312.

Luukka P (2011), Feature selection using fuzzy entropy measures similarity classifier. Expert Systems with Applications 38(4):4600-4607.

Manickavasagan A, Sathya G, Jayas DS (2008), Comparison of illuminations to identify wheat classes using monochrome images. Computers and Electronics in Agriculture 63(2):237-244.

Manickavasagan A, Al-Yahyai R (2012), Quality assessment of dates by computer vision technology. In Dates - Production, Processing, Food and Medicinal Values Ed. Manickavasagan A, Essa MM, Sukumar E, PP-217-226, Taylor \& Francis Group: New York, NY, USA.

Manickavasagan A, Al-Shekaili HN, Thomas G, Rahman MS, Guizani N, Jayas DS (2013), Edge detection features to evaluate hardness of dates using monochrome images. Food and Bioprocess Technology (DOI 10.1007/s11947-013-1219-0). 
Constrast Enhancement using Brightness Preserving Histograph Equalization Technique for Classification of Date Varieties

Otsu N (1979), A threshold selection method from gray-level histogram. IEEE Transactions on System Man Cybernetics 9(1):62-66.

Prakash OM, Sharma PK, Maharajan (2008), New measures of weighed fuxxy entropy and their applications for the study of maximum weighted fuzzy entropy principle. Information Sciences 178:2389-2395.
Shannon CE (1948), A mathematical theory of communication. Bell System Technical Journal 27(3):379-423.

Wang C, Ye Z (2005), Brightness preserving histogram equalization with maximum entropy: a variational perspective. IEEE Transactions on Consumer Electronics 51(4):1326-1334. 